\title{
Role of Exogenous Nitric Oxide Donor in Treatment of Decompensated Hemorrhagic Shock in Normotensive and Hypertensive Rats
}

\author{
Majid Khazaei ${ }^{1}$ and Babak Barmaki ${ }^{2}$ \\ ${ }^{1}$ Department of Physiology, Isfahan University of Medical Sciences, Isfahan 81746-73836, Iran \\ ${ }^{2}$ Department of Physiology, Zabol University of Medical Sciences, Zabol, Iran
}

Correspondence should be addressed to Majid Khazaei, khazaei@med.mui.ac.ir

Received 11 March 2012; Revised 7 April 2012; Accepted 21 April 2012

Academic Editor: Rudi Beyaert

Copyright (C) 2012 M. Khazaei and B. Barmaki. This is an open access article distributed under the Creative Commons Attribution License, which permits unrestricted use, distribution, and reproduction in any medium, provided the original work is properly cited.

\begin{abstract}
Introduction. In this study, we investigated the role of exogenous NO donor, sodium nitroprusside (SNP), on hemodynamic responses and survival rate during decompensated hemorrhagic shock in normotensive and hypertensive rat. Methods. Male wistar rats were divided into normotensive and hypertensive groups $(n=12$ each). Then, the animals were subjected to decompensated hemorrhagic shock by withdrawing blood until the mean arterial pressure (MAP) reached to $40 \mathrm{mmHg}$. After the shock period, the animals were randomly assigned to SNP-treated $(0.5 \mathrm{mg} / \mathrm{kg})$ and control groups $(n=6 \mathrm{each})$. MAP and heart rate (HR) were monitored throughout the experiment and $60 \mathrm{~min}$ after the administration of drug. Serum NO concentrations were measured. The survival rate was counted during next $72 \mathrm{~h}$. Results. Infusion of SNP caused no significant changes in MAP and HR in normotensive and hypertensive animals. Hemorrhagic shock increased serum NO concentration and SNP administration reduced serum NO concentration in either normotensive or hypertensive groups. Survival counts during $72 \mathrm{~h}$ after experiment did not improve by SNP administration, and there were no significant differences between normotensive and hypertensive groups. Conclusion. SNP administration cannot improve hemodynamic responses and survival count during decompensated hemorrhagic shock in normotensive and hypertensive animals.
\end{abstract}

\section{Introduction}

Hemorrhagic shock is characterized by a decrease in blood pressure, reduced cardiac output, and release of vasoconstrictor substances and in, decompensating phase, delayed vascular decompensation [1].

Several vasoactive factors have been proposed to be involved in the development of vascular hyporeactivity during shock period including nitric oxide (NO) [2-4]. Studies indicated that NO is involved in pathogenesis of shock and modulates cardiovascular responses during various types of shock [5]. NO overproduction may be involved in vascular decompensation and hyporeactivity during decompensated hemorrhagic shock $[3,6]$. Vascular hyporeactivity can interfere with vasoconstrictor agents uses in therapy of shock. On the other hand, other studies demonstrated the beneficial effects of NO donors or Larginine, NO precursor during blood loss $[7,8]$. Thus, the exact role of $\mathrm{NO}$ and $\mathrm{NO}$ donors during hemorrhagic shock is still unclear.

The initial response to blood loss is an increase to sympathetic tone and peripheral vascular resistance [1]. Hypertensive subjects have higher baseline sympathetic tone and vascular resistance. It is documented that hypertensive patients have a more severe circulatory dysfunction after hemorrhage and higher mortality rate after hemorrhage than normotensive subjects [9]. Since hypertension is associated with several cardiovascular abnormalities, in this study, we investigated the effect of sodium nitroprusside (SNP), an exogenous NO donor, on hemodynamic responses and survival rate during decompensated hemorrhagic shock in normotensive and hypertensive rats. 


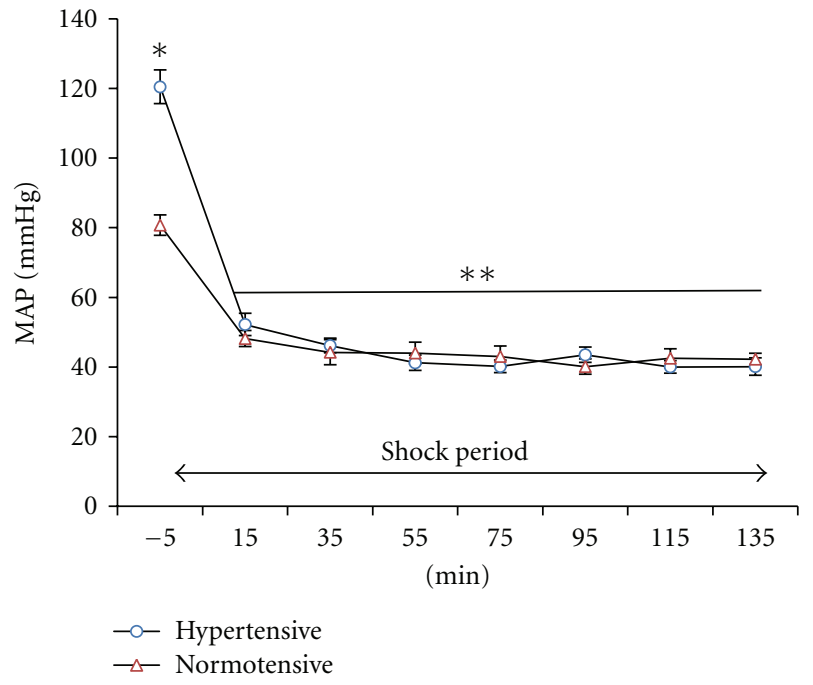

(a)

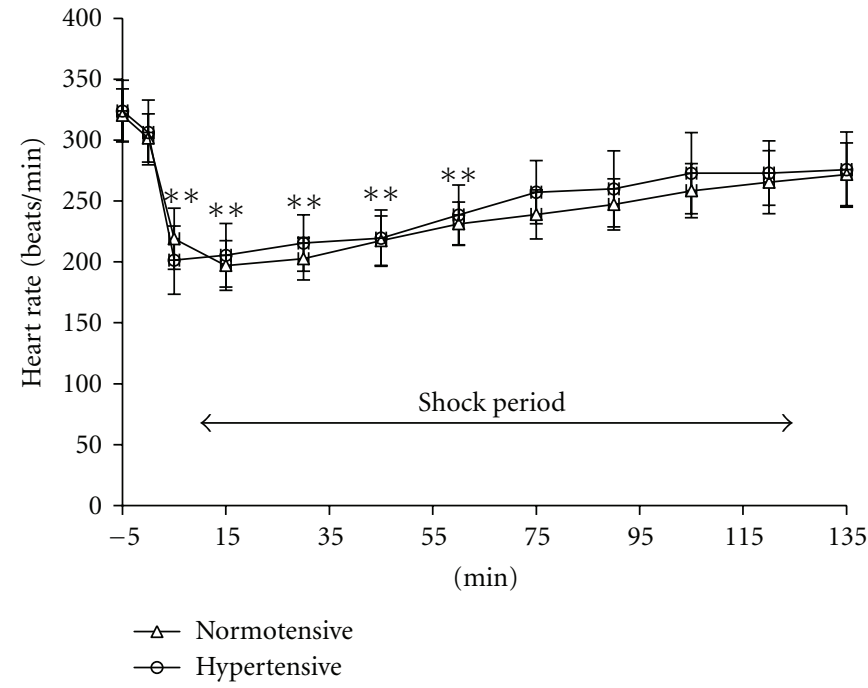

(b)

FIGURE 1: Changes of MAP (a) and HR (b) during shock period in normotensive and hypertensive rats. ${ }^{*} P<0.05$ compare to normotensive group. ${ }^{*} P<0.05$ compared to before experiment.

\section{Materials and Methods}

2.1. Animals and Experimental Groups. The experiments were performed on male Wistar rats weighting 280-320 g. The animals were acclimatized with animal room for one week and had free access to standard food and water. The rats were kept three per cages and exposed to a $12: 12 \mathrm{~h}$ lightdark cycle, temperature between 22 and $25^{\circ} \mathrm{C}$, and humidity around $60-70 \%$. All experimental procedures were approved by the ethical committee of Isfahan University of Medical Sciences.

Hemorrhagic shock was induced in all experimental groups. The groups were as follows: (1) normotensive; (2) normotensive + SNP; (3) hypertensive; (4) hypertensive + $\operatorname{SNP}(n=6$ each $)$.

2.2. DOCA-Salt Hypertensive Model. In all groups, the animals were anaesthetized by intraperitoneally injection of ketamine $(75 \mathrm{mg} / \mathrm{kg})$ and xylazine $(7.5 \mathrm{mg} / \mathrm{kg})$. An incision was made on right flank and all rats were uninephrectomized. Then, they were randomly divided into normotensive and hypertensive groups. The animals in hypertensive groups received Deoxycorticosterone Acetate (DOCA) injection $30 \mathrm{mg} / \mathrm{kg}$ (Aboureihan Co.) subcutaneously, two times a week for 8 weeks as previously described [10]. They also received $\mathrm{NaCl} 1 \%$ solution instead of tap water for drinking. The normotensive groups received tap water and injection of solvent of DOCA throughout the study. Systolic blood pressure was recorded every week by tail cuff pressure.

2.3. Decompensated Hemorrhagic Shock. After 8 weeks, the animals were anaesthetized by ketamine $(75 \mathrm{mg} / \mathrm{kg})$ and xylazine $(7.5 \mathrm{mg} / \mathrm{kg})$ and maintained under anesthesia for the duration of the experiment. The body temperature was maintained around $37^{\circ} \mathrm{C}$ using a heating pad (Harvard Apparatus, England) and monitored by rectal thermometer. Then, the right femoral artery and vein were cannulated using PE-50 catheters for blood withdrawal and drug administration, respectively. Left femoral artery was also cannulated and connected to the power lab system for monitoring the mean arterial pressure (MAP) and heart rate (HR) throughout the experiment.

After stabilizing period for 30 minutes, hemorrhagic shock was induced by withdrawing blood every 2 minutes using a $1 \mathrm{~mL}$ syringe containing $5 \mathrm{Iu}$ heparin until MAP decreases to $40 \mathrm{mmHg}$ during the total time of 15 minutes [11]. The animals were maintained to shock state for next 120 min. During this time, blood was withdrawn or reinfused, if necessary, to maintain MAP at this level. After the shock period, the animals were randomly assigned to SNPtreated and nontreated groups. SNP $(0.5 \mathrm{mg} / \mathrm{kg}$; Sigma $)$ was dissolved in $0.9 \%$ sodium chloride solution $(1 \mathrm{~mL} / \mathrm{kg})$ [12] and infused through the femoral vein catheter during $15 \mathrm{~min}$. MAP was monitored for $60 \mathrm{~min}$ after the administration of drug. Groups 1 and 3 received sodium chloride solution with the same volume.

2.4. Blood Collection. Blood samples of $1 \mathrm{~mL}$ were taken after anesthesia (before experiment), at the end of shock period (120th $\mathrm{min}$ ), and $60 \mathrm{~min}$ after treatment. The blood collected was accounted in the total volume taken for induction of hemorrhage. The blood samples were centrifuged at $3000 \mathrm{rpm}$ for $20 \mathrm{~min}$ and serums were poured in eppendorf tubes and stored at $-70^{\circ} \mathrm{C}$ until analysis.

2.5. Survival Time. At the end of experiments, the catheters were removed. The arteries and vein were ligated and 


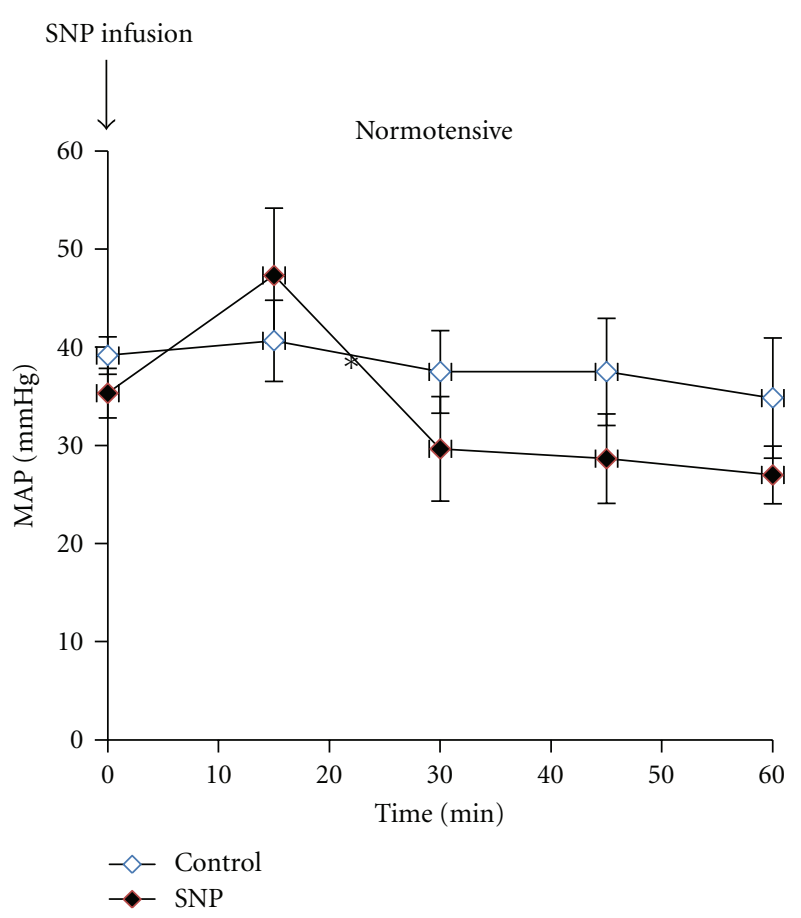

(a)

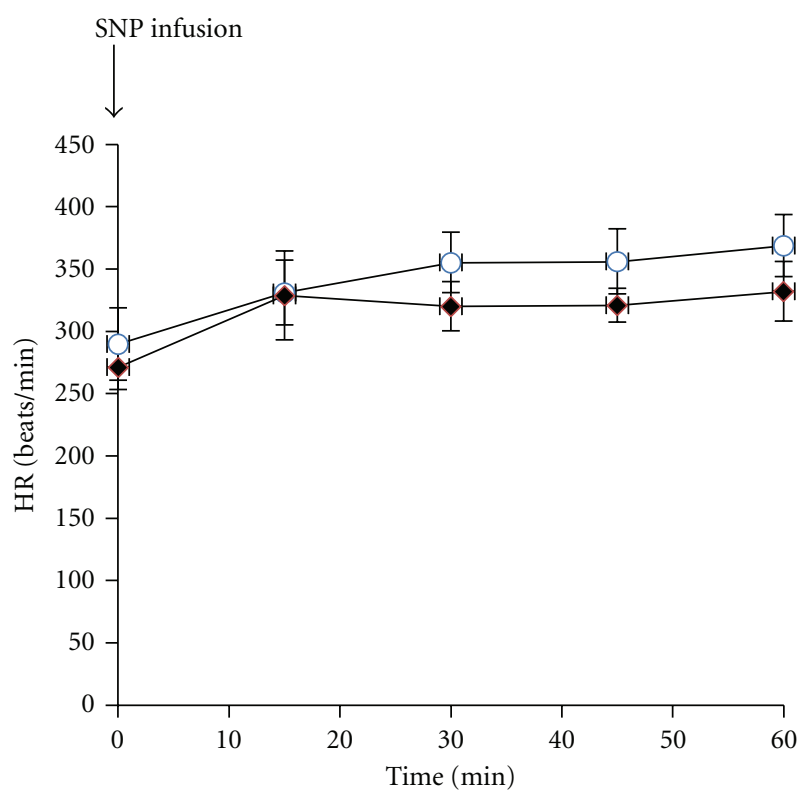

(c)

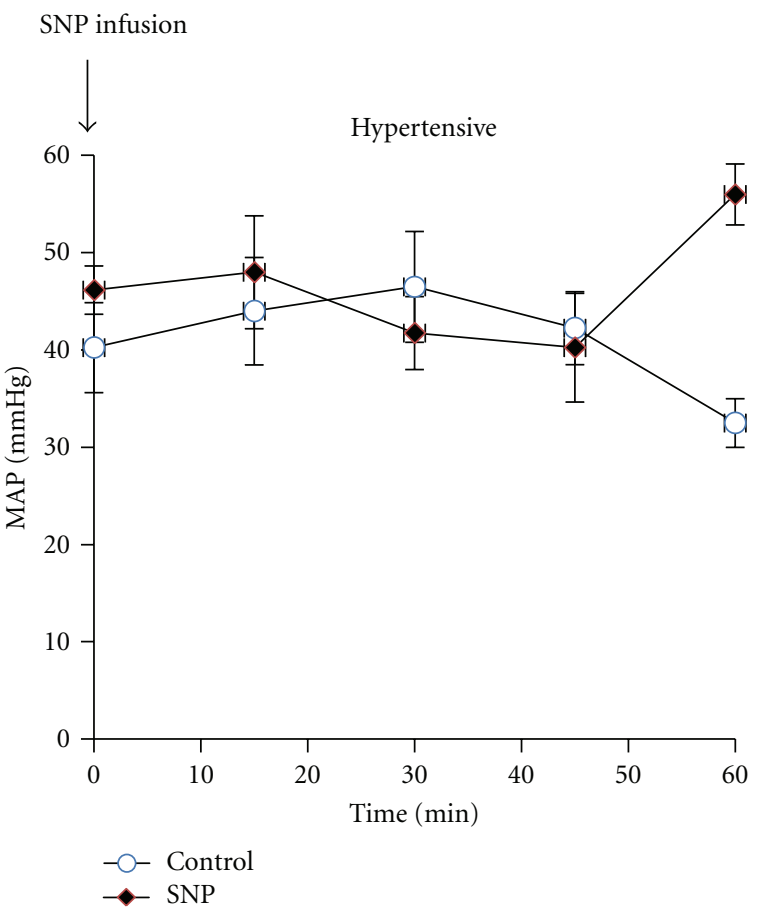

(b)

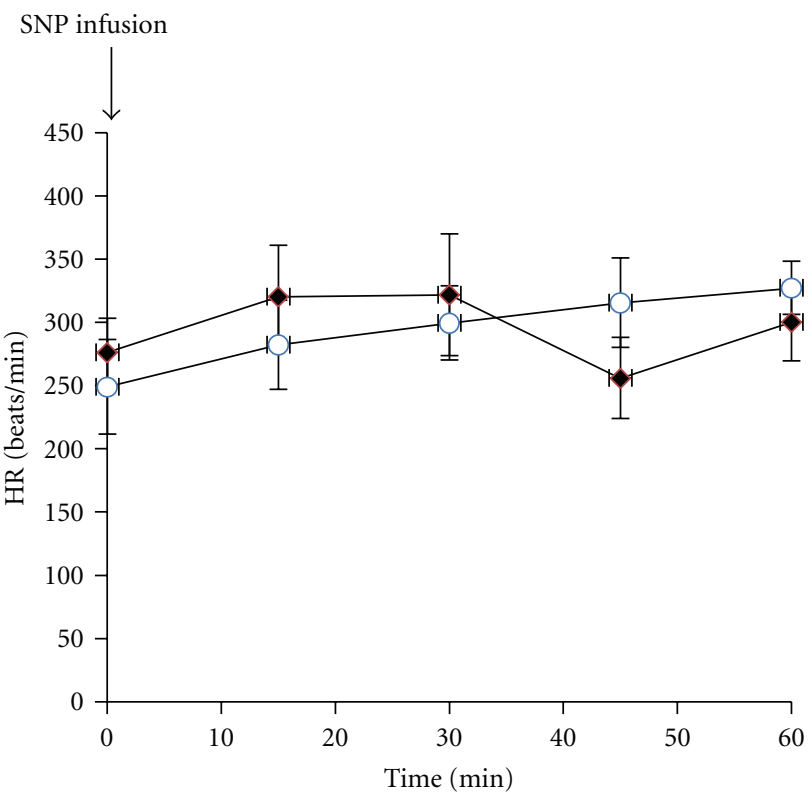

(d)

FIGURE 2: Effect of SNP administration on MAP and HR after $2 \mathrm{~h}$ hemorrhagic shock period in normotensive and hypertensive rats. ${ }^{*} P<0.05$ compared to nontreated group.

incisions closed by 3-0 chromic catgut sutures. After recovery of anesthesia, the animals were returned to individual cages and allowed free access to water and food. The survival rate was counted on first $4 \mathrm{~h}$ after experiment and every $12 \mathrm{~h}$ up to $72 \mathrm{~h}$ until the death of animals.
2.6. Serum NO Measurement. Serum NO measurement was measured using griess reagent systems as previously described [13]. In this method, the stable end metabolite of NO, nitrite, was measured. In brief, the samples were added to a 96-well enzymatic assay plate. Then, sulfanilamide solution 


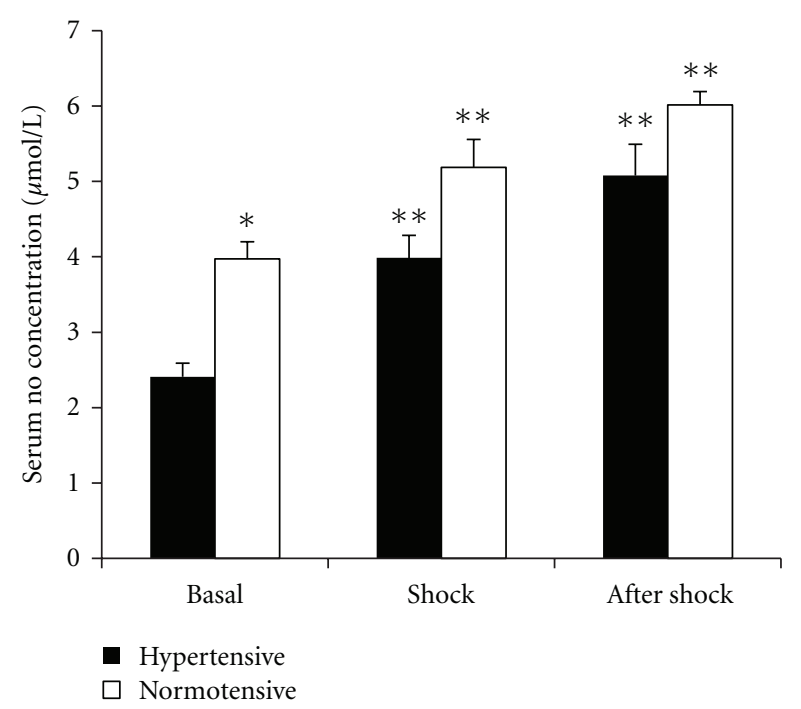

Figure 3: Serum NO concentrations at different periods in normotensive and hypertensive groups. ${ }^{*}$ Indicates significant difference compared to hypertensive group; ${ }^{* *}$ indicates significant differences compared to basal state.

and N-1-naphtylethylenediamine dihydrochloride solution were added to each well, respectively. Absorbance was measured using microplate reader at the wavelength of $520 \mathrm{~nm}$. Serum NO concentrations were determined in comparison to standard reference curve. The limit detection of this assay was $2.5 \mu \mathrm{mol}$.

2.7. Statistical Analysis. The results are expressed as mean \pm standard error. Paired $t$-test was performed to compare paired data. The differences between groups were analyzed by one-way ANOVA. Survival rate was evaluated with chisquare test. $P$ less than 0.05 was considered as significant.

\section{Results}

3.1. Hemodynamic Parameters. Baseline MAP and systolic blood pressure in DOCA-Salt hypertensive rats were significantly higher than normotensive animals (MAP: $120.43 \pm$ 4.84 versus $80.71 \pm 2.93 \mathrm{mmHg}$; systolic blood pressure: $156.64 \pm 5.33$ versus $109.32 \pm 5.69 \mathrm{mmHg}$ ). Baseline $\mathrm{HR}$ in hypertensive group was not different from normotensive group $(P>0.05)$. Changes of MAP and HR during the hemorrhagic shock and shock period are presented in Figure 1. MAP was maintained around $40 \mathrm{mmHg}$ during shock period. During this time, significant bradycardia was induced at $5 \mathrm{~min}$ following the bleeding, and then, HR gradually increased during the shock period. There were no significant differences in HR between hypertensive and normotensive groups $(P>0.05)$. SNP administration caused a transient nonsignificant increase of MAP in normotensive animals; however, after $15 \mathrm{~min}$, MAP was gradually decreased. In hypertensive animals, infusion of SNP during shock period did not alter MAP and HR (Figure 2).
3.2. Serum NO Concentration. Changes of serum NO concentrations are illustrated in Figure 3. In the basal state (before experiment), serum NO concentration in hypertensive animals was lower than normotensive group $(3.98 \pm 0.36$ versus $5.18 \pm 0.36 \mu \mathrm{mol} / \mathrm{L} ; P<0.05)$. Hemorrhage increased serum NO levels in both groups $(P<0.05)$. Administration of SNP significantly reduced serum nitrite concentration in normotensive $(3.03 \pm 0.30$ versus $5.18 \pm 0.36 \mu \mathrm{mol} / \mathrm{L})$ and hypertensive $(2.56 \pm 0.26$ versus $3.98 \pm 0.36 \mu \mathrm{mol} / \mathrm{L})$ groups $(P<0.05)$.

3.3. Survival Count. All animals were survived at the end of experimental protocol. Four of six (66\%) normotensive animals who received SNP were alive $12 \mathrm{~h}$ after experiment, while one of six normotensive animals without treatment was alive during this time. After $72 \mathrm{~h}$, there were no significant differences between SNP-treated and nontreated groups. In hypertensive animals, three of six (50\%) animals were alive $4 \mathrm{~h}$ after experiment in SNP-treated and nontreated groups. After this time, there was no significant differences in survival rate of hypertensive groups between SNP-treated and nontreated groups (Figure 4).

\section{Discussion}

Many factors including NO modulate cardiovascular responses during shock state $[3,5,14]$. NO has several effects on cardiovascular systems including modulation of synaptic signaling and regulation of blood pressure [15]. In this study, we found that serum NO concentration in hypertensive animals was lower than normotensive group. Hypertension is associated with cardiovascular abnormalities including endothelial dysfunction and it seems that reduced serum NO concentration in hypertensive group may be the result of endothelial dysfunction [16, 17]. Previous studies also supported the lower NO bioavailability in hypertensive subjects [15-17]. Higher production of reactive oxygen species, lower endothelial NO synthase expression, and/or impaired L-arginine uptake are possible mechanisms for reduced NO bioavailability in hypertensive subjects $[16,17]$.

In the present study, we showed that, during the shock period, serum NO level was increased either in normotensive or hypertensive animals. In addition, increased serum NO concentrations were continued one hour after shock period. NO has been implicated in pathogenesis of hemorrhagic shock $[3,5,6]$. Lines of evidence supported that NO produced during and after ischemia is an important factor in the pathogenesis of ischemic injury [18] and is associated with vascular decompensation due to low NO bioavailability [19]. Upregulation of inducible and endothelia forms of NO synthase expression in different organs during shock is one of the possible mechanisms of excessive NO production during hemorrhagic shock $[6,20,21]$. Higher production of NO not only may reduce responsiveness to vasoactive agents, but also may contribute to major organs ischemia [22].

We also showed that infusion of SNP (an exogenous NO donor) during the decompensated hemorrhagic shock did not significantly change MAP and HR in normotensive 


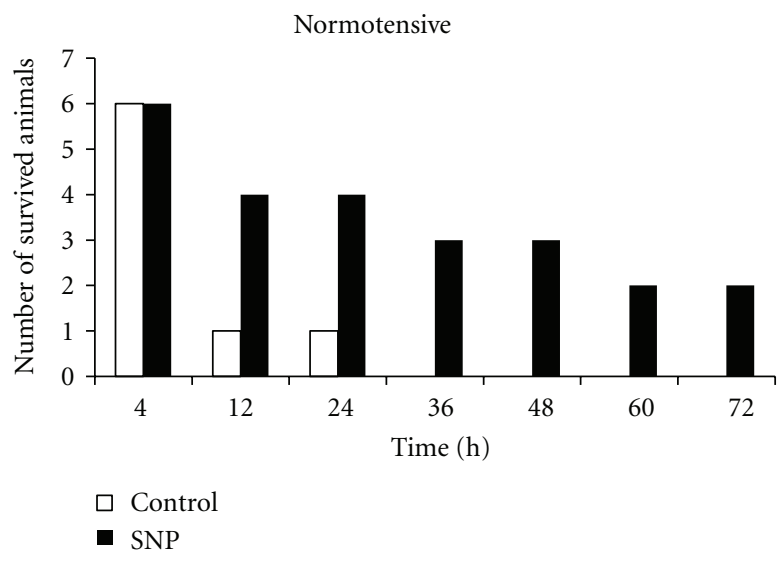

(a)

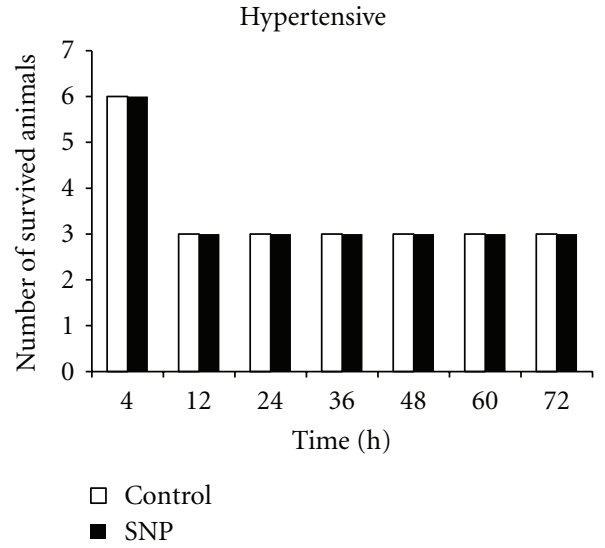

(b)

FIGURE 4: Comparison of survival rate in normotensive and hypertensive animals in SNP-treated and control groups.

and hypertensive animals. Survival rate in hypertensive animals was not affected by SNP administration, while, in normotensive group, survival rate was improved, although it was not statistically significant. Excessive production of NO may induce vascular hyporesponsiveness during shock $[3,6]$. Furthermore, it can increase reactive oxygen species and PGE2 which might be involved in organ damages during shock $[11,15]$. On the other hand, elevation of NO improves microcirculation and tissue perfusion [15]. Thus, the exact role of NO during various types of shock is still unclear. Several studies have been done to indicate the role of NO donors in various shock models; however, the results are contradictory. Intravenous injection of L-arginine, the precursor of NO, in hemorrhagic shocked rats attenuated lung injury associated with hemorrhage [7]. A recent study by Arora et al. showed that administration of L-arginine in severe hemorrhaged rats improved outcome, functional and metabolic, and suggested that these benefits are related to local perfusion and inflammation [8]. Exogenous NO also prevents cardiovascular decompensation, maintained the heart rate [23], and preserved microvascular perfusion [19]. In agreement with our results, a recent study by Sobhian et al. showed that in the rats subjected to hemorrhage, NOsupplemented resuscitation improves gastrointestinal blood flow and despite a transient MAP reduction, survival rate was not affected [24]. Shirhan et al. also demonstrated that SNP infusion during refractory hemorrhagic shock although served as protective in early stage of hemorrhagic shock, but did not provide protection in the later period [11]. In the present study, we found that SNP administration during decompensated shock hemorrhage did not improve hemodynamic response and survival rate. Although, in this study, we did not determine organ blood flow, however, it seems that, despite improved tissue perfusion after NO donors, it cannot be of protective effects on hemodynamic responses and survival count in normotensive animals, although, based on our knowledge, no study has been done to define the role of NO donors during hemorrhaged shock in hypertensive subjects.
In conclusion, it seems that although SNP administration may improve organ flow and preserve microcirculation, however, it cannot improve hemodynamic response and survival count in normotensive and hypertensive animals.

\section{Acknowledgments}

The study was supported by a Grant from Isfahan University of Medical Sciences (Grant no. 387413). The authors thank Aboureihan Co. for supplying DOCA.

\section{References}

[1] R. F. Bond and G. Johnson, "Vascular adrenergic interactions during hemorrhagic shock," Federation Proceedings, vol. 44, no. 2, pp. 281-289, 1985.

[2] B. Zingarelli, F. Squadrito, D. Altavilla, G. Calapai, M. di Rosa, and A. P. Caputi, "Role of tumor necrosis factor- $\alpha$ in acute hypovolemic hemorrhagic shock in rats," American Journal of Physiology, vol. 266, no. 4, pp. H1512-H1515, 1994.

[3] L. M. Liu, J. A. Ward, and M. A. Dubick, "Hemorrhageinduced vascular hyporeactivity to norepinephrine in select vasculatures of rats and the roles of nitric oxide and endothelin," Shock, vol. 19, no. 3, pp. 208-214, 2003.

[4] S. Li, S. X. Fan, and T. M. McKenna, "Role of nitric oxide in sepsis-induced hyporeactivity in isolated rat lungs," Shock, vol. 5, no. 2, pp. 122-129, 1996.

[5] A. M. Balaszczuk, N. D. Arreche, M. McLaughlin, C. Arranz, and A. L. Fellet, "Nitric oxide synthases are involved in the modulation of cardiovascular adaptation in hemorrhaged rats," Vascular Pharmacology, vol. 44, no. 6, pp. 417-426, 2006.

[6] L. M. Liu and M. A. Dubick, "Hemorrhagic shock-induced vascular hyporeactivity in the rat: relationship to gene expression of nitric oxide synthase, endothelin-1, and select cytokines in corresponding organs," Journal of Surgical Research, vol. 125, no. 2, pp. 128-136, 2005.

[7] G. Preissler, F. Löhe, U. Ebersberger et al., "Recipient treatment with L-Arginine attenuates donor lung injury associated with hemorrhagic shock," Transplantation, vol. 87, no. 11, pp. 1602-1608, 2009. 
[8] T. K. Arora, A. K. Malhotra, R. Ivatury, and M. J. Mangino, "LArginine infusion during resuscitation for hemorrhagic shock: impact and mechanism," The Journal of Trauma and Acute Care Surgery, vol. 72, no. 2, pp. 397-402, 2012.

[9] Z. Radisavljevic, "Hypertension-induced dysfunction of circulation in hemorrhagic shock," American Journal of Hypertension, vol. 8, no. 7, pp. 761-767, 1995.

[10] B. Seifi, M. Kadkhodaee, S. M. Karimian, M. Zahmatkesh, J. $\mathrm{Xu}$, and $\mathrm{M}$. Soleimani, "Evaluation of renal oxidative stress in the development of doca-salt induced hypertension and its renal damage," Clinical and Experimental Hypertension, vol. 32, no. 2, pp. 90-97, 2010.

[11] M. D. Shirhan, S. M. Moochhala, K. L. Siew Yang et al., "The role of selective nitric oxide synthase inhibitor on nitric oxide and $\mathrm{PGE}_{2}$ levels in refractory hemorrhagic-shocked rats," Journal of Surgical Research, vol. 123, no. 2, pp. 206-214, 2005.

[12] R. Anaya-Prado, L. H. Toledo-Pereyra, J. Walsh, R. F. Guo, J. Reuben, and P. A. Ward, "Exogenous nitric oxide donor and related compounds protect against lung inflammatory response after hemorrhagic shock and resuscitation," Journal of Trauma, vol. 57, no. 5, pp. 980-988, 2004.

[13] M. Nematbakhsh and M. Khazaei, "The effect of estrogen on serum nitric oxide concentrations in normotensive and DOCA Salt hypertensive ovariectomized rats," Clinica Chimica Acta, vol. 344, no. 1-2, pp. 53-57, 2004.

[14] A. Yaghi, N. A. M. Paterson, and D. G. McCormack, "Vascular reactivity in sepsis: importance of controls and role of nitric oxide," American Journal of Respiratory and Critical Care Medicine, vol. 151, no. 3, pp. 706-712, 1995.

[15] U. Forstermann, "Nitric oxide and oxidative stress in vascular disease," Pflügers Archiv, vol. 459, no. 6, pp. 923-939, 2010.

[16] E. Gkaliagkousi, S. Douma, C. Zamboulis, and A. Ferro, "Nitric oxide dysfunction in vascular endothelium and platelets: role in essential hypertension," Journal of Hypertension, vol. 27, no. 12, pp. 2310-2320, 2009.

[17] A. S. Levy, J. C. S. Chung, J. T. Kroetsch, and J. W. E. Rush, "Nitric oxide and coronary vascular endothelium adaptations in hypertension," Vascular Health and Risk Management, vol. 5, pp. 1075-1087, 2009.

[18] L. Phillips, A. H. Toledo, F. Lopez-Neblina, R. Anaya-Prado, and L. H. Toledo-Pereyra, "Nitric oxide mechanism of protection in ischemia and reperfusion injury," Journal of Investigative Surgery, vol. 22, no. 1, pp. 46-55, 2009.

[19] P. Cabrales, A. G. Tsai, and M. Intaglietta, "Exogenous nitric oxide induces protection during hemorrhagic shock," Resuscitation, vol. 80, no. 6, pp. 707-712, 2009.

[20] R. Anaya-Prado, L. H. Toledo-Pereyra, R. F. Guo, J. Reuben, P. A. Ward, and J. Walsh, "The attenuation of hemorrhageinduced liver injury by exogenous nitric oxide, L-Arginine, and inhibition of inducible nitric oxide synthase," Journal of Investigative Surgery, vol. 16, no. 5, pp. 247-261, 2003.

[21] M. McDonald, M. Abdelrahman, S. Cuzzocrea, and C. Thiemermann, "Tyrphostin reduces the organ injury in haemorrhagic shock: role of inducible nitric oxide synthase," Resuscitation, vol. 58, no. 3, pp. 349-361, 2003.

[22] M. Shirhan, S. M. Moochhala, S. Y. L. Kerwin, K. C. Ng, and J. $\mathrm{Lu}$, "Influence of selective nitric oxide synthetase inhibitor for treatment of refractory haemorrhagic shock," Resuscitation, vol. 61, no. 2, pp. 221-229, 2004.

[23] P. Nachuraju, A. J. Friedman, J. M. Friedman, and P. Cabrales, "Exogenous nitric oxide prevents cardiovascular collapse during hemorrhagic shock," Resuscitation, vol. 82, no. 5, pp. 607613,2011 .
[24] B. Sobhian, M. Jafarmadar, H. Redl, and S. Bahrami, "Nitric oxidesupplemented resuscitation improves early gastrointestinal blood flow in rats subjected to hemorrhagic shock without late consequences," American Journal of Surgery, vol. 201, no. 1, pp. 100-110, 2011. 


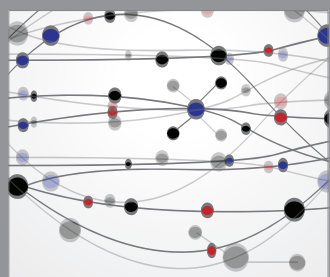

The Scientific World Journal
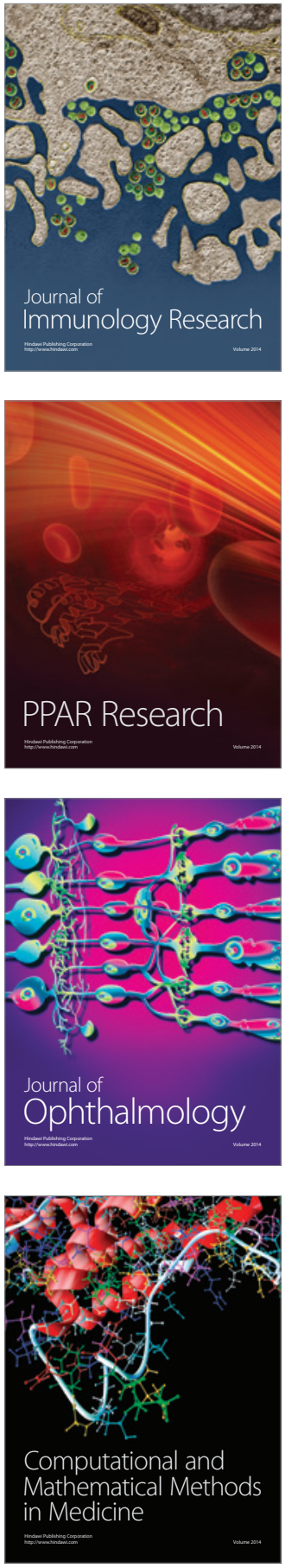

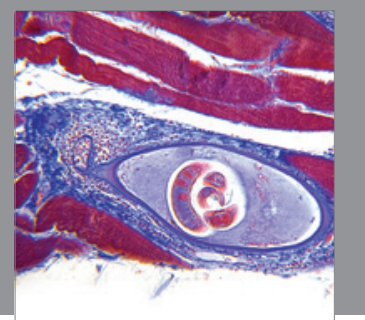

Gastroenterology

Research and Practice
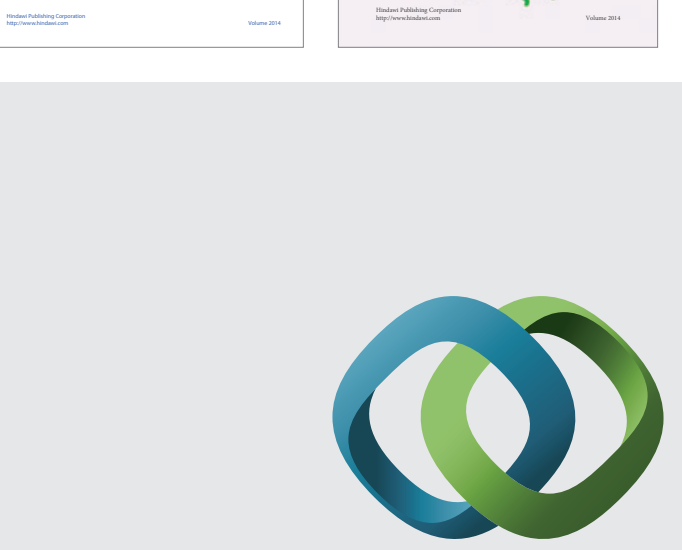

\section{Hindawi}

Submit your manuscripts at

http://www.hindawi.com
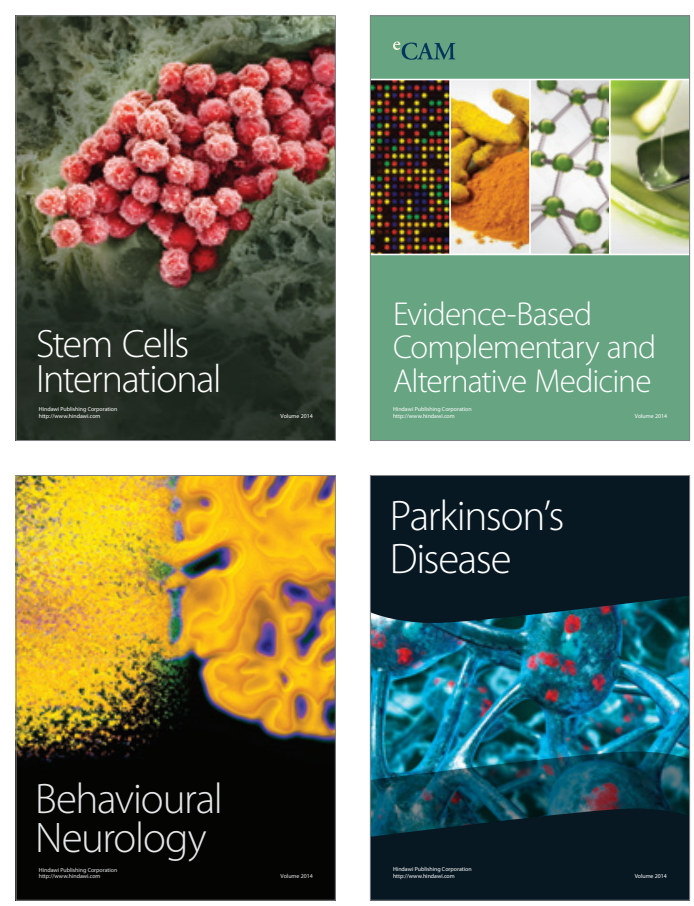

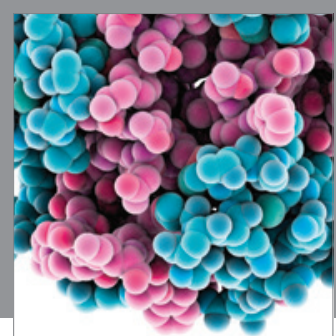

Journal of
Diabetes Research

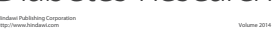

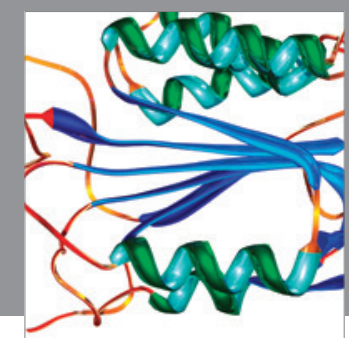

Disease Markers
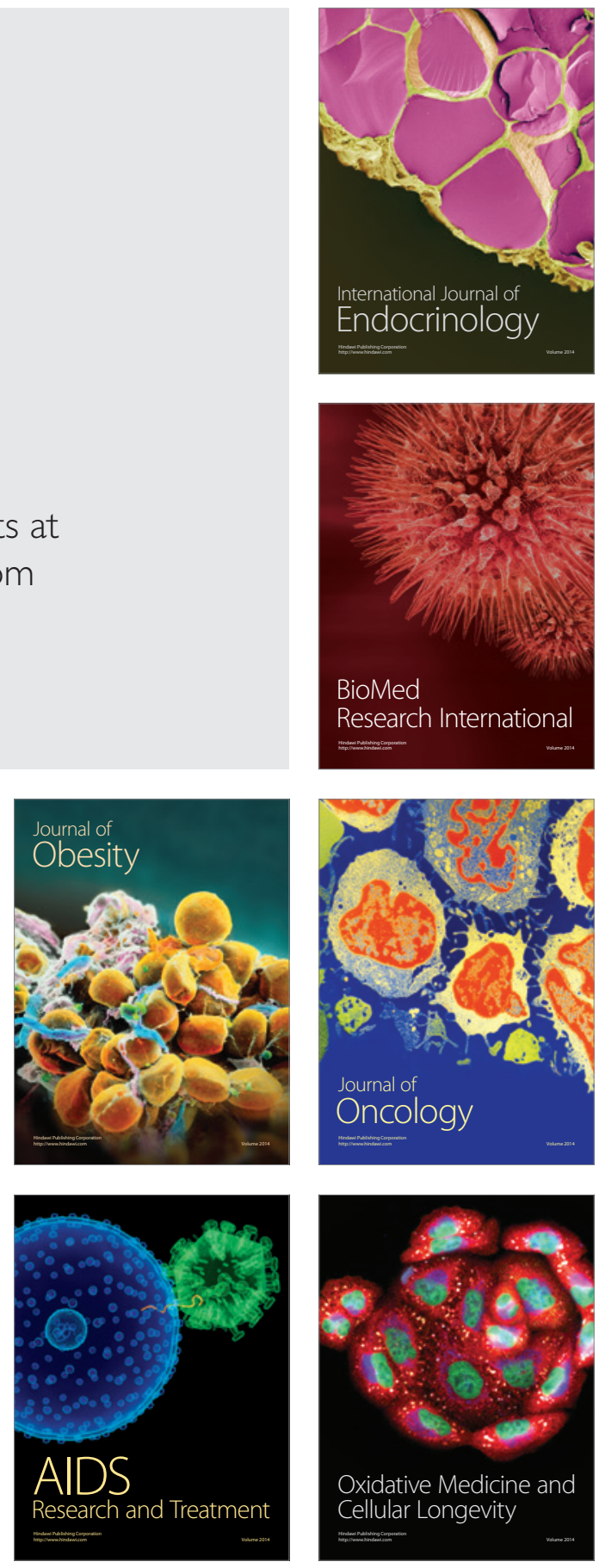